\title{
Multifractality Tests Using Bootstrapped Wavelet Leaders
}

\author{
Herwig Wendt, Member, IEEE, and Patrice Abry
}

\begin{abstract}
Multifractal analysis, which mostly consists of measuring scaling exponents, is becoming a standard technique available in most empirical data analysis toolboxes. Making use of the most recent theoretical results, it is based here on the estimation of the cumulants of the log of the wavelet Leaders, an elaboration on the wavelet coefficients. These log-cumulants theoretically enable discrimination between mono- and multifractal processes, as well as between simple log-normal multifractal models and more advanced ones. The goal of the present contribution is to design nonparametric bootstrap hypothesis tests aiming at testing the nature of the multifractal properties of stochastic processes and empirical data. Bootstrap issues together with six declinations of test designs are analyzed. Their statistical performance (significances, powers, and p-values) are assessed and compared by means of Monte Carlo simulations performed on synthetic stochastic processes whose multifractal properties (and log-cumulants) are known theoretically a priori. We demonstrate that the joint use of wavelet Leaders, log-cumulants, and bootstrap procedures enable us to obtain a powerful tool for testing the multifractal properties of data. This tool is practically effective and can be applied to a single observation of data with finite length.
\end{abstract}

Index Terms-Bootstrap, hypothesis test, multifractal analysis, wavelet leaders.

\section{Motivation}

$\mathbf{S}$ CALING or multifractal (MF) analyses [1], [2] nowadays belong to most standard empirical data analysis toolboxes. Scaling, or scale invariance, is indeed a property that has been extensively observed in empirical data produced from numerous applications of very different nature such as turbulence, network traffic and biomedical signals.

Stating that some data $X$ possess scaling properties mostly amounts to assuming and checking that its structure functions $S(q, a)$ behave as power laws of the analysis scale $a$, for a given range of scales $a \in\left[a_{m}, a_{M}\right], a_{M} / a_{m} \gg 1$ and for a given range of statistical orders $q$, usually including $q=2$ :

$$
S(q, a)=\frac{1}{n_{a}} \sum_{k=1}^{n_{a}}\left|T_{X}(a, k)\right|^{q} \simeq G_{q} a^{\zeta(q)} .
$$

Here, the $T_{X}(a, t)$ stand for multiresolution quantities such as the wavelet coefficients, $n_{a} \approx n / a$ denotes the number of such coefficients available at each scale $a$, while $n$ stands for the observation duration (the $G_{q}$ depend on the details of the process $X$ and are functions of the variable $q$, but not of the analysis

Manuscript received August 28, 2006; revised February 1, 2007. The associate editor coordinating the review of this manuscript and approving it for publication was Prof. Leslie Collins.

The authors are with CNRS and the Laboratoire de Physique, Ecole Normale Supérieure de Lyon, 69364 Lyon, France (e-mail: herwig.wendt@ens-lyon.fr; patrice.abry@ens-lyon.fr).

Digital Object Identifier 10.1109/TSP.2007.896269 scale a). Empirical multifractal analysis essentially consists of estimating the scaling exponents $\zeta(q)$ from a given set of data. These scaling exponents are commonly involved in various data analysis tasks, such as detection, identification, or classification.

The function $\zeta(q)$ can formally be expanded as a polynomial in $q: \zeta(q)=c_{1} q+c_{2} q^{2} / 2+c_{3} q^{3} / 6+\cdots$. When $\zeta(q)$ reduces to a linear function of $q, X$ is said to be monofractal. Self-similar processes such as fractional Brownian motion (FBM) constitute a celebrated and widely used class of monofractal processes. In the present contribution, $X$ is said to be multifractal when $\zeta(q)$ departs from a linear behavior in $q \cdot{ }^{1}$ In the case of $\zeta(q)=c_{1} q+c_{2} q^{2} / 2$, the simplest departure from linear, $X$ is referred to as a log-normal multifractal (LN-MF) process. This process represents the most, if not only, practically used multifractal model. More complex multifractal models, such as compound Poisson cascades (CPC), (cf. e.g., [3]), could theoretically be used and involve polynomials $\zeta(q)$ of order higher than 2. Therefore, the estimation of the precise values of the $c_{p}$ 's is crucial for practical purposes. Mainly, deciding on whether $c_{2}=0$, or whether $c_{3}=0$ when $c_{2} \neq 0$, is central to select which model (FBM, LN-MF, or higher order MF) best describes the data.

The goal of the present contribution is to propose statistical tests aiming at deciding whether $p \geq 2, c_{p}=0$ or not and thus at discriminating between mono- and multifractal processes. So far, this issue, which is commonly mentioned as being essential to empirical multifractal analysis, received no systematic or detailed study. The hypothesis tests proposed here rely on the combination of the three key ingredients log-cumulants, wavelet Leaders, and nonparametric bootstrap:

First, it has been shown recently [4], [5] that a relevant multifractal formalism should be based on wavelet Leaders rather than on wavelet coefficients [1], [2]. For instance, wavelet Leaders-based multifractal estimation procedures significantly outperform those based on wavelet coefficients [6]. Wavelet coefficients and Leaders are fully defined in Section II-A. A brief review of multifractal analysis and formalisms is given in Section II-B.

Second, an alternative to multifractal estimation procedures based on (1) has been proposed originally during the early 1990s in [7] and further developed in [8]: From estimations of the cumulants of the logarithm of the multiresolution quantities, the coefficients $c_{p}$ of the polynomial expansion of $\zeta(q)$ can be estimated directly. As explained above, these so-called $l o g$ cumulants are of particular interest, since they naturally emphasize the difference between mono- and multifractal processes. Log-cumulant expansions are introduced in Section II-C.

\footnotetext{
${ }^{1}$ A rigorous mathematical definition of mono- versus multifractality remains an involved mathematical issue and is further addressed in Section II-B.
} 
Third, we use nonparametric bootstrap techniques for the design of the statistical tests. Bootstrap was introduced during the 1980s [9] and has recently regained interest due to continuously growing computer facilities [10]-[12]. It consists of approximating an unknown distribution of a random variable by means of repeated resampling with replacement from the available data. The use of bootstrap techniques in the wavelet domain was first reported in [13]. Bootstrap has also been considered for the estimation of the Hurst parameter of self-similar processes [14], and for the estimation of scaling exponents and log-cumulants for both mono - and multifractal processes [6], [15]. In the present work, we use nonparametric bootstrap methods on wavelet Leaders and coefficients as robust means for obtaining approximate null distributions of test statistics for hypothesis tests on $c_{p}$. Six declinations for the precise construction of the empirical acceptance region are analyzed and compared. Basics on hypothesis tests and non parametric bootstrap tests, together with the definitions of the acceptance regions are detailed in Section III-B. In order to assess the statistical performance (significances, $\mathrm{p}$-values, and powers) of the proposed bootstrap tests, large sets of Monte Carlo (MC) simulations are performed. The corresponding methodology, the simulation setup, as well as the multifractal processes used to conduct the numerical simulations, are presented in Section IV. The results show that the bootstrap tests exhibit satisfactory performance and are reported and discussed in Section V. We end up with a robust and powerful practical test procedure for the analysis of a single and finite-length observation of empirical data. This is detailed in Section VI, together with conclusions and perspectives.

\section{WAVELETS AND MULTIFRACTAL ANALYSIS}

\section{A. Wavelet Coefficients and Wavelet Leaders}

Wavelet Coefficients: Let $\psi_{0}(t)$ denote a reference pattern whose energy remains mostly concentrated in a narrow support both in the time and frequency domains. This function $\psi_{0}(t)$ is commonly referred to as the mother wavelet and can be further characterized by its number of vanishing moments, a strictly positive integer $N_{\psi} \geq 1$ defined as $\forall k=0,1, \ldots, N_{\psi}$ $1, \int_{\mathcal{R}} t^{k} \psi_{0}(t) d t \equiv 0$ and $\int_{\mathcal{R}} t^{N_{\psi}} \psi_{0}(t) d t \neq 0$. Let $\left\{\psi_{j, k}(t)=\right.$ $\left.2^{-j} \psi_{0}\left(2^{-j} t-k\right), j \in \mathcal{N}, k \in \mathcal{N}\right\}$ denote the collection of templates of $\psi_{0}$, dilated to scales $a=2^{j}$, and translated to time positions $2^{j} k$. Let us further assume that the $\left\{\psi_{j, k}(t), j \in \mathcal{N}, k \in\right.$ $\mathcal{N}\}$ forms an orthonormal basis of $L^{2}(\mathbb{R})$. Let $X(t), t \in[0, n)$ denote the process under analysis and $n$ its observation duration. The wavelet coefficients of $X$ are obtained as comparisons, by means of inner products: $d_{X}(j, k)=\left\langle\psi_{j, k} \mid X\right\rangle$. The $d_{X}(j, k)$ therefore provide a time-scale representation of $X$ that fully characterizes it: $X(t)=\sum_{j, k} d_{X}(j, k) \psi_{j, k}(t)$. For a detailed introduction to wavelet transforms, the reader is referred to, e.g., [16] and [17].

Wavelet Leaders: Let us now further assume that $\psi_{0}(t)$ has a compact time support and let us introduce the indexing $\lambda_{j, k}=$ $\left[k 2^{j},(k+1) 2^{j}\right)$ and the union $3 \lambda_{j, k}=\lambda_{j, k-1} \cup \lambda_{j, k} \cup \lambda_{j, k+1}$. The wavelet Leaders $L_{X}(j, k)$ are defined as

$$
L_{X}(j, k)=\sup _{\lambda^{\prime} \subset 3 \lambda_{j, k}}\left|d_{\lambda^{\prime}}\right|
$$

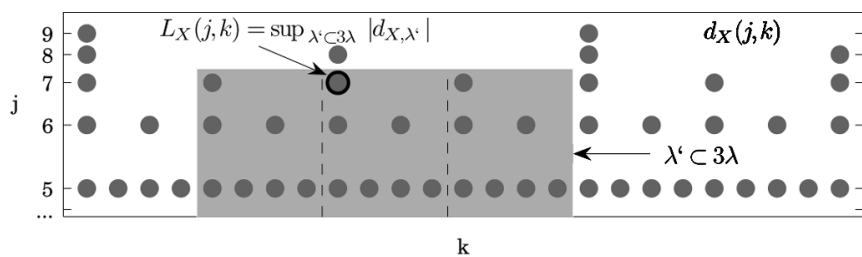

Fig. 1. Wavelet Leaders $L_{X}$ (black circle) are calculated from the discrete wavelet coefficients $d_{X}(\cdot, \cdot)$ (dots) by taking the supremum in the time neighborhood $3 \lambda_{j, k}$, over all finer scales $2^{j^{\prime}}<2^{j}$ (area in gray).

where the supremum is taken on the discrete wavelet coefficients $d_{X}(\cdot, \cdot)$ in the time neighborhood $3 \lambda_{j, k}$ over all finer scales $2^{j^{\prime}}<2^{j}$. Fig. 1 illustrates this definition.

\section{B. Multifractal Analysis}

Multifractal Spectrum: Multifractal analysis aims at characterizing the signal $X$ under analysis through the description of the variations along time of the regularity of its sample path. Such a local regularity is measured by means of Hölder exponents $h(t)$. The Hölder exponent quantifies the strength of the singular behavior of $X$ around $t_{0}$, by comparing the local variations of $X$ around $t_{0}$ to a local power law behavior: $X\left(t_{0}\right)$ is said to belong to $C^{\alpha}\left(t_{0}\right)$ with $\alpha \geq 0$ if there exists a constant $C>0$ and a polynomial $P_{t_{0}}(t)$ with $\operatorname{deg}\left(P_{t_{0}}\right)<\alpha$ such that

$$
\left|X(t)-P_{t_{0}}(t)\right| \leq C\left|t-t_{0}\right|^{\alpha} .
$$

The Hölder exponent is defined as the largest $\operatorname{such} \alpha$, as follows:

$$
h\left(t_{0}\right)=\sup \left\{\alpha: X \in C^{\alpha}\left(t_{0}\right)\right\} .
$$

Instead of making use of a function of time $h(t)$, it is usually preferred to describe the variability of the range of Hölder exponents actually encountered in $X$ through the multifractal (or singularity) spectrum $D(h)$, which is defined as the Hausdorff dimensions of the sets of points $t_{i}$ for which $h\left(t_{i}\right)=h$.

Multifractal Formalisms: Therefore, empirical or practical multifractal analysis mostly consists of inferring $D(h)$ from a single finite duration observation of data: this is commonly referred to as a multifractal formalism. In a nutshell, multifractal formalisms essentially amount first to performing estimations of the scaling exponents $\zeta(q)$ (by direct use of (1)) and second to relating those estimates to $D(h)$ via a Legendre transform.

For a long period of time, wavelet coefficients have been considered as the key quantities empirical multifractal analysis should be based on [1], [4], [18]-[21]. However, recent results [4], [5] show that this wavelet-based multifractal formalism suffers from two major drawbacks: It does not enable us to reach the entire multifractal spectrum of the process under analysis, and it is not valid for all types of multifractal processes. Notably, processes containing oscillating singularities are incorrectly analyzed.

Leader-Based Multifractal Formalism: A more relevant multifractal formalism holds if wavelet coefficients are replaced with the wavelet Leaders, defined above. By construction, Leaders are monotonously increasing with scale $2^{j}$, a property that has been recently shown to be key in designing a multifractal formalism [4]. Indeed, under mild regularity conditions 
on the sample path, wavelet Leaders exactly reproduce the Hölder exponent of $X(t)$ at $t_{0}$, i.e., $h\left(t_{0}\right)$ is the supremum of all values $h$ such that, in the limit of fine scales $\left(2^{j} \rightarrow 0\right)$,

$$
L_{X}(j, k) \leq C 2^{j h}
$$

Following intuitions originally developed in [22] making use of an increment-based multifractal formalism, (5) above suggests that the wavelet Leader structure functions $S^{L}\left(q, 2^{j}\right)$ possess power law behavior with respect to scales in the limit $2^{j} \rightarrow 0$ :

$$
S^{L}\left(q, 2^{j}\right)=\frac{1}{n_{j}} \sum_{k=1}^{n_{j}} L_{X}(j, k)^{q}=\left.\left.F_{q}\right|^{j}\right|^{\zeta(q)} .
$$

Here, $n_{j} \approx n / 2^{j}$ is the number of Leaders available at each scale and $n$ the length of the sample. Under mild uniform Hölder regularity condition on $X(t)$, it has been shown [4], [23] that (6) is an exact result and that the Legendre transform of the $\zeta(q)$ provides a tight upper bound for the multifractal spectrum $D(h)$ :

$$
D(h) \leq \min _{q \neq 0}(1+q h-\zeta(q)) .
$$

It turns out that for most, if not all, commonly used multiplicative processes (whose multifractal spectra are concave), the inequality (7) is an equality and thus relates the scaling exponents to the multifractal spectrum.

For more thorough introductions to multifractal analysis, the reader is referred to, e.g., [4], [20], [23], and [24].

Multi- Versus Monofractal: Rigorous mathematical definitions of monofractality and multifractality refer to whether the characterization of $X(t)$ requires a single Hölder exponent or a collection of different such exponents. In the present contribution, we refer to monofractal (respectively, multifractal) processes when their scaling exponents $\zeta(q)$ follow a linear behavior in $q$ (respectively, depart from a linear behavior in $q$ ). This definition is not rigorously true and results in a little loss of generality: It excludes specific processes such as Lévy stable self-similar processes or random wavelet series which are of theoretical interest but remain difficult to use in applications. This restrictive definition of monofractality is formulated by analogy with finite variance self-similar processes with stationary increments, which are widely used in applications and is sufficient for most practical purposes.

\section{Log-Cumulants}

For some classes of multifractal processes [7], [25], (6) takes a more general form

$$
\mathbb{E} L_{X}(j, \cdot)^{q}=F_{q}\left|2^{j}\right|^{\zeta(q)} .
$$

Using the second characteristic functions of the distributions of the random variables $\ln L_{X}(j, \cdot)$, (8) can be rewritten as

$$
\ln \mathbb{E} e^{q \ln L_{X}(j, \cdot)}=\sum_{p=1}^{\infty} C_{p}^{j} \frac{q^{p}}{p !}=\ln F_{q}+\zeta(q) \ln 2^{j}
$$

where the $C_{p}^{j}$ stand for the cumulants of order $p \geq 1$ of $\ln L_{X}(j, \cdot)$. Equation (9) implies that the $C_{p}^{j}$,s must satisfy

$$
\forall p \geq 1: C_{p}^{j}=c_{p}^{0}+c_{p} \ln 2^{j}
$$

and therefore that

$$
\ln \mathbb{E} e^{q \ln L_{X}(j, \cdot)}=\underbrace{\sum_{p=1}^{\infty} c_{p}^{0} \frac{q^{p}}{p !}}_{\ln F_{q}}+\underbrace{\sum_{p=1}^{\infty} c_{p} \frac{q^{p}}{p !}}_{\zeta(q)} \ln 2^{j}
$$

where $c_{p}^{0}$ and $c_{p}$ do not depend on the scale $2^{j}$. This yields

$$
\zeta(q)=\sum_{p=1}^{\infty} c_{p} \frac{q^{p}}{p !} .
$$

Thus, the measurements of the scaling exponents $\zeta(q)$ can be interestingly replaced by those of the log-cumulants $c_{p}$.

The main benefit of this change of multifractal attributes lies in the fact that the $c_{p}$ 's emphasize the difference between scaling exponents $\zeta(q)$ that are linear in $q$ (equivalently, $\forall p \geq 2: c_{p} \equiv$ $0)$ and $\zeta(q)$ that depart from linear (equivalently, there exists $p \geq 2: c_{p} \neq 0$ ) [7], [25]. In other words, according to our previous definitions, knowing whether $c_{2}=0$ or not is practically equivalent to choosing between mono- versus multifractality. Also, the $c_{p}$ 's enable to discriminate between simple LN-MF processes $\left(p \geq 3: c_{p}=0\right)$ and more complex multifractal models, such as CPCs $\left(c_{3} \neq 0\right)$. Therefore, in the sequel of this contribution, we concentrate on estimating the $c_{p}$ and on testing whether $c_{p}=0$ or not.

\section{Estimation Procedures}

Estimations of the Cumulants $C_{p}^{j}$ : From the $n$ samples of $X$, one computes $n_{j} \approx n / 2^{j}$ wavelet Leaders $L_{X}(j, k)$ at each scale $j$. The asymptotically unbiased and consistent standard estimators (e.g., [26]) are used to obtain the estimations $\hat{C}_{p}^{j}$ of the cumulants of $\ln L_{X}(j, k)$.

Linear Regressions-log-Cumulants $c_{p}$ : Based on (10), the $c_{p}$ 's are estimated by linear regressions of $\hat{C}_{p}^{j}$ versus $\ln 2^{j}$ :

$$
\hat{c}_{p}=\log _{2} e \sum_{j=j_{1}}^{j_{2}} w_{j} \hat{C}_{p}^{j}
$$

where $\left(j_{1}, j_{2}\right)$ defines the range of scales, $2^{j_{1}} \leq a \leq 2^{j_{2}}$, over which the linear regressions are performed.

Weights: The weights $w_{j}$ in (12) have to satisfy the usual constraints $\sum_{j_{1}}^{j_{2}} j w_{j} \equiv 1$ and $\sum_{j_{1}}^{j_{2}} w_{j} \equiv 0$. A standard form reads $w_{j}=\left(1 / b_{j}\right)\left(S_{0} j-S_{1}\right) /\left(S_{0} S_{2}-S_{1}^{2}\right)$, with $S_{i}=\sum_{j_{1}}^{j_{2}} j^{i} / b_{j}, i=0,1,2$. The freely selectable positive numbers $b_{j}$ reflect the confidence granted to each $\hat{C}_{p}^{j}$. In the present work, we select $b_{j}=1 / n_{j}$ (cf. [1]), without loss of generality.

Coefficients Versus Leaders: The estimation procedures presented above involve wavelet Leaders $L_{X}(j, k)$. Note that corresponding procedures can be based on wavelet coefficients, $m u$ tatis mutandis. The same is true for all test procedures described 
in the next section. The respective performance of Leader and coefficient-based test procedures are compared in Section V.

\section{Testing Statistical Hypothesis ON LOG-Cumulants}

\section{A. Statement of the Problem}

We want to test $c_{p}=c_{p, 0}$ against the two-sided alternative $c_{p} \neq c_{p, 0}$. Eventually, the specific case $c_{2,0}=0$ is seen as the test of mono- versus multifractality.

Given a single sample of $X$ of size $n$, the $d_{X}(j, k)$ 's, $L_{X}(j, k)$ 's, $\hat{C}_{p}^{j}$, and $\hat{c}_{p}$ are computed and estimated according to the procedures described in Section II-D. We denote the unknown distributions of $X, \mathcal{L}_{X}(j,),. \hat{C}_{p}^{j}$ and $\hat{c}_{p}$ by $F_{\boldsymbol{\theta}_{\chi}}^{\chi}, \chi=\left\{X, \mathcal{L}_{j}, C_{p}^{j}, c_{p}\right\}$ with parameters $\boldsymbol{\theta}_{\chi}$, respectively. The distributions $F_{\boldsymbol{\theta}_{\chi}}^{\chi}$ are unknown members of families of distributions $F_{\boldsymbol{\Theta}^{\prime}}^{\chi}, \boldsymbol{\theta}_{\chi} \in \boldsymbol{\Theta}_{\chi}$.

We consider the basic test statistic

$$
\begin{aligned}
T_{B} & =c_{p}-c_{p, 0} \\
\hat{t}_{B} & =\hat{c}_{p}-c_{p, 0}
\end{aligned}
$$

and the studentized test statistic

$$
\begin{aligned}
& T_{S}=\frac{c_{p}-c_{p, 0}}{\sigma} \\
& \hat{t}_{S}=\frac{\hat{c}_{p}-c_{p, 0}}{\hat{\sigma}^{*}}
\end{aligned}
$$

where $\sigma$ and $\hat{\sigma}^{*}$ stand for the theoretical standard deviation of $\hat{c}_{p}$ and for its bootstrap estimation (cf. (22) below), respectively. The studentized test statistic attempts to make the random variable $\hat{t}_{S}$ pivotal, i.e., to remove the unknown parameter $\sigma$ from the distribution of $\hat{c}_{p}$.

\section{B. Statistical Tests}

1) Definition: Tests for such problems can be constructed in the following way (see, e.g., [27]): A null hypothesis

$$
H_{0}: c_{p}=c_{p, 0} ; \quad \text { equivalently } \quad H_{0}: \boldsymbol{\theta}_{\chi} \in \boldsymbol{\Theta}_{\chi, 0}
$$

postulates that $\boldsymbol{\theta}_{\chi}$ is a member of a specified subset $\boldsymbol{\Theta}_{\chi, 0} \subset \boldsymbol{\Theta}_{\chi}$ of the set of all possible parameter vectors. $H_{0}$ is simple if it completely specifies all the parameters describing the family of distributions $F_{\Theta_{\chi}}^{\chi}$, i.e., the subset $\Theta_{\chi, 0}=\left\{\boldsymbol{\theta}_{\chi, 0}\right\}$ contains only one single element. Otherwise, $H_{0}$ is composite.

For $H_{0}$ simple, the null distribution of $T$ is given by

$$
P_{\boldsymbol{\theta}_{\chi, 0}}^{T}(\tau)=\operatorname{Pr}\left\{T \leq \tau \mid F_{\boldsymbol{\theta}_{\chi, 0}}\right\}
$$

and a $(1-\alpha)$ acceptance region $\mathcal{T}_{(1-\alpha)}$ can be defined as a set on the real axis for which

$$
\operatorname{Pr}\left\{T \in \mathcal{T}_{(1-\alpha)} \mid P_{\boldsymbol{\theta}_{\chi, 0}}^{T}\right\}=1-\alpha .
$$

For instance, this could be the equitailed interval $\mathcal{T}_{(1-\alpha)}=$ $\left[t_{\alpha / 2}, t_{1-\alpha / 2}\right]$, where $t_{\alpha}$ denotes the $\alpha$ quantile of the null distribution (14). The complement of $\mathcal{T}_{(1-\alpha)}$ is called the rejection region. The test $d_{\alpha}$ is then

$$
d_{\alpha}= \begin{cases}1 & \text { if } \hat{t} \notin \mathcal{T}_{(1-\alpha)} \\ 0 & \text { otherwise }\end{cases}
$$

Thus, $d_{\alpha}$ rejects $H_{0}$ if, for a given preset value $\alpha$, the observed value $\hat{t}$ of the test statistic is in the rejection region.

2) Significance and p-Value: The quantity $\alpha$ is called the level or significance of the test and equals the error rate in rejecting $H_{0}$.

The critical value of $\alpha$ for which the observed test statistic $\hat{t}$ would be regarded as just decisive against $H_{0}$ is called the $p$-value or significance $p$ of $\hat{t}$

$$
p=\left(\alpha \mid d_{\alpha}=0, d_{\alpha+\varepsilon}=1\right), \quad \varepsilon>0 .
$$

Under $H_{0}$, the p-value ideally has a uniform distribution on $[0,1]$, yielding its interpretation as an error rate: If $\hat{t}$ were regarded as just decisive against $H_{0}$, then this is equivalent to a procedure that rejects $H_{0}$ with error rate $p$ [12].

3) Power: When performing a test, we may commit two types of errors: First, we may reject $H_{0}$ when it is true (error of the first kind), which ideally happens with probability equal to the significance $\alpha$. Second, we may accept $H_{0}$ when it is false (error of the second kind), i.e., accept when $\left\{\boldsymbol{\theta}_{\chi} \in \boldsymbol{\Theta}_{\chi, A}\right\}$, where $\boldsymbol{\Theta}_{\chi, A}$ is the subset of alternatives, $\boldsymbol{\Theta}_{\chi, 0} \cup \boldsymbol{\Theta}_{\chi, A}=\boldsymbol{\Theta}_{\chi}$. The probability of rejection, evaluated for a given alternative $\boldsymbol{\theta}_{\chi, A} \in \boldsymbol{\Theta}_{\chi, A}$ is called the power of the test against this alternative

$$
\beta\left(\boldsymbol{\theta}_{\chi, A}, \alpha\right)=\operatorname{Pr}\left\{d_{\alpha}=1 \mid F_{\boldsymbol{\theta}_{\chi, A}}\right\}, \boldsymbol{\theta}_{\chi, A} \in \boldsymbol{\Theta}_{\chi, A} .
$$

A good test should have small $\alpha$ and large $\beta$, which are antagonistic goals. It is common practice to preset the significance $\alpha$ and then select a test with power $\beta$ as large as possible.

4) Composite Null Hypotheses: In most parametric and all nonparametric problems, $H_{0}$ is composite. This is also the case for the tests considered here. Then, the null distributions $F_{\boldsymbol{\Theta}_{\chi, 0}}$ and $P_{\boldsymbol{\Theta}_{\chi, 0}}^{T}$ are not completely specified, since $\boldsymbol{\Theta}_{\chi, 0}$ has more than one element (and possibly infinitely many elements). Therefore, the acceptance and rejection regions and the $p$-value are not well defined.

An approximate solution that is appropriate for the problem considered here is to estimate a distribution $P_{0}^{T} \in P_{\Theta_{\chi, 0}}^{T}$ that satisfies $H_{0}$ and to use this null model to define an acceptance region $\widehat{\mathcal{T}}_{(1-\alpha)}$

$$
\operatorname{Pr}\left\{T \in \widehat{\mathcal{T}}_{(1-\alpha)} \mid P_{0}^{T}\right\}=1-\alpha .
$$

The test is then given by (15), with $\widehat{\mathcal{T}}_{(1-\alpha)}$ replacing $\mathcal{T}_{(1-\alpha)}$.

\section{Nonparametric Bootstrap Tests}

1) Nonparametric Bootstrap: In order to define an acceptance region, cf. (18), for our problem, we need to estimate $P_{0}^{T} \in P_{\boldsymbol{\Theta}_{\chi, 0}}^{T}$. The distributions $P_{\Theta_{\chi, 0}}^{T}$ are, however, unknown, since the distributions $F_{\Theta_{\chi, 0}}^{\chi}$ are not known. Nonparametric bootstrap solutions to this problem consist of replacing the unknown distributions $F_{\boldsymbol{\Theta}_{\chi, 0}}^{\chi}$ by the empirical distributions $\hat{F}_{\boldsymbol{\Theta}_{\chi}}^{\chi}$, given by the samples $\chi=\left\{X, \mathcal{L}_{j}, C_{p}^{j}\right\}$. The bootstrap estimations of $P_{0}^{T} \in P_{\boldsymbol{\Theta}_{T, 0}}$ are

$$
\hat{P}_{0}^{T}(\tau)=\operatorname{Pr}\left\{\hat{t}^{*} \leq \tau \mid \hat{F}_{\boldsymbol{\theta}_{\chi}}^{\chi}\right\}
$$


with $\hat{t}^{*}$ :

$$
\begin{aligned}
\hat{t}_{B}^{*} & =\hat{c}_{p}^{*}-\hat{c}_{p} \\
\hat{t}_{S}^{*} & =\frac{\hat{c}_{p}^{*}-\hat{c}_{p}}{\hat{\sigma}^{* *}} .
\end{aligned}
$$

The $\hat{c}_{p}^{*}$ s and $\hat{\sigma}^{* *}$ are, respectively, bootstrap estimations for $c_{p}$ (cf. (20) below) and for the standard deviations of $\hat{c}_{p}^{*}$ [cf. (23), below]. Since the empirical distributions $\hat{F}_{\boldsymbol{\theta}_{\chi}}^{\chi}$ do not necessarily satisfy $H_{0}, c_{p, 0}$ must be replaced by $\hat{c}_{p}$ in the bootstrap versions $\hat{t}_{B}^{*}$ and $\hat{t}_{S}^{*}$ of the original test statistics $\hat{t}_{B}$ and $\hat{t}_{S}$. This ensures that $\hat{P}_{0}^{T}$ approximately satisfies $H_{0}$ [28].

2) Resampling Procedure: Equation (19) is solved through simulation. We fix the empirical distribution $\hat{F}_{\boldsymbol{\theta}_{\chi}}^{\chi}$ to be the samples of Leaders, $\hat{F}_{\boldsymbol{\theta}_{\mathcal{L}_{j}}}^{\mathcal{L}_{j}}$. This has been shown to perform well in the context of wavelet-based multifractal analysis (see [6] and [13]-[15]). Despite the decorrelating property of the wavelet transform, the width of the time support of the wavelet introduces some very short term correlation among the samples $\mathcal{L}_{j}$. Thus, we use a moving blocks bootstrap with block length equal to the (finite) size of the time support of the wavelet (cf. [6]). For Daubechies wavelets used here, it amounts to $B=2 \cdot N_{\psi}$. At each scale $a=2^{j}, R$ bootstrap resamples $\mathcal{L}_{j}^{*(1)}, \ldots, \mathcal{L}_{j}^{*(R)}$ are generated from the original samples $\mathcal{L}_{j}=\left\{L_{X}(j, 1), \ldots, L_{X}\left(j, n_{j}\right)\right\}$. Each resample $\mathcal{L}_{j}^{*}=\left\{L_{X}^{*(j, 1)}(\cdot), \ldots, L_{X}^{*\left(j, n_{j}\right)}(\cdot)\right\}$ is an unsorted collection of $n_{j}$ sample points, drawn blockwise and with replacement from the original sample $\mathcal{L}_{j}$.

3) Bootstrap Estimations: The collections $\mathcal{L}_{j}^{*(r)}$ are used to compute $R$ bootstrap cumulant estimations $\left\{\hat{C}_{p}^{j *(r)}\right\}_{r=1}^{R}$. From these, we obtain the $R$ log-cumulant bootstrap estimations, as follows:

$$
\hat{c}_{p}^{*(r)}=\log _{2} e \sum_{j=j_{1}}^{j_{2}} w_{j} \hat{C}_{p}^{j *(r)}
$$

and the bootstrap test statistics $\left\{\hat{t}_{B}^{*(r)}\right\}_{r=1}^{R}$ and $\left\{\hat{t}_{S}^{*(r)}\right\}_{r=1}^{R}$. Finally, the empirical bootstrap distributions

$$
\hat{P}_{0}^{T}(\tau) \approx \frac{1+\sum_{r=1}^{R} H\left(\tau-\hat{t}^{*(r)}\right)}{R+1}
$$

approximate (19), where $H(x)=1$ when $x \geq 0$ and 0 elsewhere. These distributions are used to determine approximate limits of the acceptance regions $\widehat{\mathcal{T}}_{(1-\alpha)}$ (see Section III-C-5), necessary for performing the test (15) and for estimating the p-value (16). A typical bootstrap distribution, obtained for $c_{2}$ (for a LN-MF process, cf. Section IV-B) and the basic bootstrap test procedure are illustrated in Fig. 2.

4) Standard Deviation Estimations for Studentized Statistic $T_{s}$ : The bootstrap estimations $\hat{\sigma}^{*}$ are readily obtained from the samples $\hat{c}_{p}$ ( $\widehat{\text { Std }}$ denotes the sample standard deviation):

$$
\hat{\sigma}^{*}=\widehat{\operatorname{Std}} \hat{c}_{p}^{*} \approx \operatorname{Std} \hat{c}_{p} .
$$

For obtaining estimations $\hat{\sigma}^{* *(r)}$ of the standard deviation of each $\hat{c}_{p}^{*(r)}$, a second layer of bootstrap resampling is necessary on top of the first one: From each bootstrap resample $\mathcal{L}_{j}^{*(r)}, S$

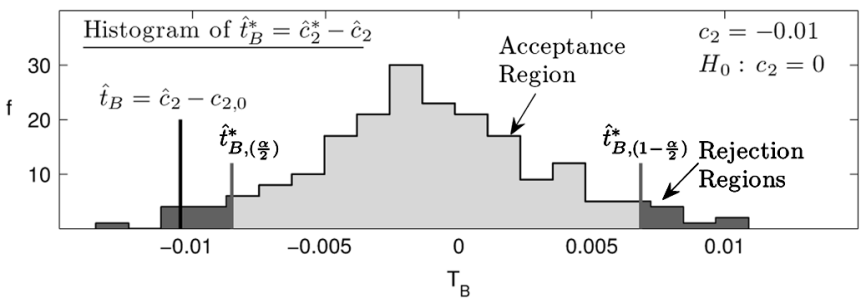

Fig. 2. Estimated null distribution and $(1-\alpha)$ basic bootstrap test for the second log-cumulant of MRW (single realization): The hypothesis $H_{0}$ is rejected if the observed value $\hat{t}$ of the test statistic is within the rejection regions, i.e., outside the interval $\widehat{\mathcal{T}}_{(1-\alpha)}^{\text {bas }}=\left[\hat{t}_{(\alpha) /(2)}^{*}, \hat{t}_{1-(\alpha) /(2)}^{*}\right]$.

double bootstrap resamples $\mathcal{L}_{j}^{* *(r, s)}$ are obtained as an unsorted collection of $n_{j}$ sample points, drawn blockwise and with replacement from $\mathcal{L}_{j}^{*(r)}$, similar to the procedure described above. These collections are used to compute the $R \cdot S$ double bootstrap estimations $\hat{c}_{p}^{* *(r, s)}, \hat{t}^{* *(r, s)}$, and

$$
\hat{\sigma}^{* *(r)}=\widehat{\operatorname{Std}} \hat{c}_{p}^{* *(r, \cdot)} \approx \operatorname{Std} \hat{c}_{p}^{*(r)} .
$$

5) Bootstrap Test Acceptance Regions: As we consider tests against double-sided alternatives, double-sided acceptance regions $\mathcal{T}_{(1-\alpha)}$ are used in the present work, that is, acceptance regions with finite lower and upper limits. There exists a large number of nonparametric bootstrap tests in the literature, producing different acceptance regions (cf. [12] for an overview). We analyze six different significance $\alpha$ bootstrap tests, including simple, computationally cheap, and more sophisticated, computationally expensive bootstrap methods: The asymptotic bootstrap test uses simple symmetric acceptance regions, employing only the bootstrap standard deviation estimations. The basic and percentile tests employ quantiles of the empirical bootstrap distributions (21) of $\hat{t}_{B}^{*}$. The studentized test uses quantiles of the empirical distributions of the pivoted test statistic $\hat{t}_{S}^{*}$. The adjusted basic and adjusted percentile tests use the double bootstrap estimations to correct for a bias in the limits of the acceptance regions of the basic and percentile tests. The three latter methods are potentially more performant, however at the price of a costly double bootstrap layer.

Asymptotic (Normal) Bootstrap Test: Assuming $T_{B}$ to be approximately normal, the bootstrap standard deviation estimation $\hat{\sigma}^{*}$ is used to construct the equitailed and symmetric acceptance region

$$
\widehat{\mathcal{T}}_{(1-\alpha)}^{\text {nor }}=\left[q_{\left(\frac{\alpha}{2}\right)} \hat{\sigma}^{*}+c_{p, 0},-q_{\left(\frac{\alpha}{2}\right)} \hat{\sigma}^{*}+c_{p, 0}\right]
$$

where $q_{\alpha}$ is the $\alpha$ quantile of the standard normal distribution.

Basic Bootstrap Test: The bootstrap distribution (21) of $\hat{t}_{B}^{*}$ is used directly to define the equitailed acceptance region

$$
\widehat{\mathcal{T}}_{(1-\alpha)}^{\text {bas }}=\left[\hat{t}_{B,\left(\frac{\alpha}{2}\right)}^{*}, \hat{t}_{B,\left(1-\frac{\alpha}{2}\right)}^{*}\right]
$$

where $\hat{t}_{B,(\alpha)}^{*}$ is the empirical $\alpha$-quantile of (21) for $\hat{t}_{B}^{*}$.

Percentile Bootstrap Test: There is a duality between significance tests for parameters and confidence sets for those parameters, in the sense that-for a prescribed level-a confidence region includes parameters that are not rejected by an appropriate 
TABLE I

OVERVIEW OF SIGNIFICANCE $\alpha$ BOOTSTRAP TESTS AND THEIR CORRESPONDING ACCEPTANCE REGIONS

$$
\begin{aligned}
& d_{\alpha}=\left\{\begin{array}{ll}
1 & \text { if } \hat{t} \notin \widehat{\mathcal{T}}_{(1-\alpha)} \\
0 & \text { otherwise. }
\end{array} \quad p=\left(\alpha \mid d_{\alpha}=0, d_{\alpha+\varepsilon}=1\right), \varepsilon>0\right. \\
& \text { Normal: } \quad \widehat{\mathcal{T}}_{(1-\alpha)}^{\text {nor }}=\left[q_{\left(\frac{\alpha}{2}\right)} \hat{\sigma}^{*}+c_{p, 0},-q_{\left(\frac{\alpha}{2}\right)} \hat{\sigma}^{*}+c_{p, 0}\right] \\
& \text { Basic: } \quad \widehat{\mathcal{T}}_{(1-\alpha)}^{\text {bas }}=\left[\hat{t}_{B,\left(\frac{\alpha}{2}\right)}^{*}, \hat{t}_{B,\left(1-\frac{\alpha}{2}\right)}^{*}\right] \\
& \text { Percentile: } \quad \widehat{\mathcal{T}}_{(1-\alpha)}^{\text {per }}=\left[-\hat{t}_{\left(1-\frac{\alpha}{2}\right)}^{*},-\hat{t}_{\left(\frac{\alpha}{2}\right)}^{*}\right] \\
& \text { Studentized: } \quad \widehat{\mathcal{T}}_{(1-\alpha)}^{s t u}=\left[\hat{t}_{S,\left(\frac{\alpha}{2}\right)}^{*}, \hat{t}_{S,\left(1-\frac{\alpha}{2}\right)}^{*}\right] \\
& \text { Adjusted Basic: } \quad \widehat{\mathcal{T}}_{(1-\alpha)}^{a d j b a s}=\left\{t: p_{a d j}^{b a s} \geq \alpha\right\} \\
& \text { Adjusted Percentile: } \quad \widehat{\mathcal{T}}_{(1-\alpha)}^{\text {adjper }}=\left\{t: p_{a d j}^{\text {per }} \geq \alpha\right\}
\end{aligned}
$$

significance test [12]. The percentile test is constructed by inversion of a percentile confidence interval $\left[\hat{c}_{p,(\alpha / 2)}^{*}, \hat{c}_{p,(1-\alpha / 2)}^{*}\right]$ for $c_{p}$ and has acceptance region

$$
\widehat{\mathcal{T}}_{(1-\alpha)}^{\text {per }}=\left[-\hat{t}_{\left(1-\frac{\alpha}{2}\right)}^{*},-\hat{t}_{\left(\frac{\alpha}{2}\right)}^{*}\right] .
$$

Studentized Bootstrap Test: The studentized test is a basic bootstrap test for the pivoted test statistic $T_{S}$. The method thus demands a double bootstrap for calculating the standard deviation estimations $\hat{\sigma}^{* *}$ and has acceptance region

$$
\widehat{\mathcal{T}}_{(1-\alpha)}^{\mathrm{stu}}=\left[\hat{t}_{S,\left(\frac{\alpha}{2}\right)}^{*}, \hat{t}_{S,\left(1-\frac{\alpha}{2}\right)}^{*}\right] .
$$

Adjusted p-Value for Basic Bootstrap Test: If the usual error rate interpretation of $p$ is to be valid, the p-value must be uniformly distributed on $[0,1]$ under $H_{0}$. This is, however, not guaranteed for composite null hypotheses and approximate null models (21). The adjusted p-value method aims at estimating an improved p-value that is more nearly uniformly distributed than the unadjusted one. It treats $p$ as the observed test statistic and estimates its distribution by resampling under the null model [12]. The double-sided adjusted p-value is

$$
p_{\text {adj }}^{\text {bas }}=2 \min \left(\operatorname{Pr}\left\{p^{\text {bas* }} \leq p^{\text {bas }} \mid \hat{F}_{\theta}\right\} ; \operatorname{Pr}\left\{p^{\text {bas* }}>p^{\text {bas }} \mid \hat{F}_{\theta}\right\}\right) .
$$

Here, $p^{\text {bas }}$ is the $\mathrm{p}$-value of the basic bootstrap test, and the $p^{\text {bas* }}$ are its bootstrap resamples, obtained through a double bootstrap. The acceptance region is

$$
\widehat{\mathcal{T}}_{(1-\alpha)}^{\text {adjbas }}=\left\{t: p_{\text {adj }}^{\text {bas }} \geq \alpha\right\}
$$

Adjusted p-value for Percentile Bootstrap Test: The adjusted $\mathrm{p}$-value for the percentile bootstrap test is given by (28) by replacing $p^{\text {bas }}$ and $p^{\text {bas* }}$ with the $\mathrm{p}$-value of the percentile bootstrap test $p^{\text {per }}$ and its corresponding bootstrap resamples $p^{\text {per* }}$, respectively. It has acceptance region

$$
\widehat{\mathcal{T}}_{(1-\alpha)}^{\text {adjper }}=\left\{t: p_{\text {adj }}^{\text {per }} \geq \alpha\right\}
$$

The bootstrap tests, $\mathrm{p}$-values and acceptance regions considered in this work are summarized in Table I.

\section{Statistical Performance Assessment}

\section{A. Methodology: Monte Carlo Simulations}

We evaluate the statistical performance of the proposed bootstrap test procedures by applying them to a large number $N_{\mathrm{MC}}$ of realizations of two different synthetic stochastic processes with a priori known and controlled multifractal properties and $\log$-cumulant values $c_{p}$. For each realization, each test procedure defined above provides us with two outputs: The decision $\hat{d}_{\alpha}$, and the p-value of the observed test statistic. From averages over realizations, we evaluate the actual significances, $p$-values and powers of the tests and compare them both against theoretical targets and against each other. The aim of this numerical study is to address the following issues: Do the bootstrap test procedures described above exhibit satisfactory statistical performance? Should one prefer wavelet coefficients or wavelet Leaders for testing mono- versus multifractality? What precise design of the acceptance region ((24)-(27)) yields the best statistical performance?

\section{B. Scaling Processes}

We make use of two stochastic processes, fractional Brownian motion (FBM) and multifractal random walk (MRW), chosen because they provide us with simple yet representative examples of Gaussian monofractal processes and non-Gaussian multifractal processes, respectively.

FBM is the only Gaussian exactly self-similar process with stationary increments. Its full definition as well as that of selfsimilarity can be found in, e.g., [29]. The statistical properties of FBM are entirely determined by the parameter $H$. FBM possesses scaling properties as in ((6)-(8)), with $\zeta(q)=q H$, for $q \in(-\infty, \infty)$. Thus, $c_{1}=H$ and $c_{p} \equiv 0$ for all $p \geq 2$.

MRW is a specific example of a LN-MF process. MRW has been introduced in [8] as a simple multifractal (hence, non-Gaussian) process with stationary increments: $X(k)=\sum_{k=1}^{n} G_{H}(k) e^{\omega(k)}$, where $G_{H}(k)$ consists of the increments of FBM with parameter $H$. The process $\omega$ is independent of $G_{H}$, Gaussian, with the following specific covariance: $\operatorname{cov}\left(\omega\left(k_{1}\right), \omega\left(k_{2}\right)\right)=\lambda \ln \left((L) /\left(\left|k_{1}-k_{2}\right|+1\right)\right)$ when $\left|k_{1}-k_{2}\right|<L$, and 0 otherwise. MRW has interesting scaling properties as in ((6)-(8)) for $q \in[-\sqrt{2 / \lambda}, \sqrt{2 / \lambda}]$, with $\zeta(q)=(H+\lambda) q-\lambda^{2} q^{2} / 2$. Hence, $c_{1}=H+\lambda, c_{2}=-\lambda^{2}$ and $c_{p} \equiv 0$ for all $p \geq 3$, and the departure from a linear behavior in $q$ is fully controlled by $c_{2}$.

\section{Simulation Setup}

The results presented here are obtained using Daubechies wavelets with $N_{\psi}=3$ vanishing moments. The sample sizes read $n=2^{J}$ with $J=\{12,15\}$, and the linear regressions (12) are performed over the scales defined by $j_{1}=3$ to $j_{2}=$ $J-4$. The bootstrap parameters are fixed to $B=2 \cdot N_{\psi}=$ $6,(R=599, S=50)$ for $N=2^{12}$ and $(R=399, S=25)$ for $N=2^{15}$. Nominal significances were chosen to be $\alpha=$ $\{0.05,0.1,0.15,0.2,0.25\}$. The process parameters are set to $H=c_{1}=0.8$ for FBM, and $(H, \lambda)=(0.72, \sqrt{0.08})$, i.e., $c_{1}=0.8$ and $c_{2}=-0.08$ for MRW. For the simulations of 
TABLE II

ACTUAL SigNIFICANCES $\hat{\alpha}_{\mathrm{MC}}$ OF BOOTSTRAP TESTS ON $c_{2}$, USING COEFFICIENTS AND LEADERS, FOR FBM $\left(c_{2,0}=c_{2}=0\right)$ AND MRW $\left(c_{2,0}=c_{2}=-0.08\right)$. NOMINAL SIGNIFICANCES AND RESUlTs Closest to Nominal VALUES ARE MARKED IN BOLD

$c_{2}$ FBM $\mid$ Coefficients $\mathbf{n}=\mathbf{2}^{12} \quad \mid$ Coefficients $\mathbf{n}=\mathbf{2}^{15}$

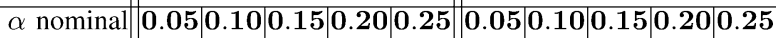

\begin{tabular}{r||c|c|c|c|c||c|c|c|c|c|}
\hline Normal & 0.10 & 0.18 & 0.23 & 0.29 & 0.35 & 0.07 & 0.13 & 0.18 & 0.24 & 0.29 \\
\hline
\end{tabular} \begin{tabular}{|l|l|l|l|l|l|l|l|l|l|l|l|l|} 
Basic & 0.08 & 0.16 & 0.23 & 0.30 & 0.34 & 0.07 & 0.13 & 0.18 & 0.23 & 0.29 \\
\hline
\end{tabular} \begin{tabular}{|l|l|l|l|l|l|l|l|l|l|l|}
\hline Percentile & 0.12 & 0.19 & 0.26 & 0.31 & 0.37 & 0.08 & $\mathbf{0 . 1 2}$ & 0.18 & 0.24 & 0.30 \\
\hline
\end{tabular}

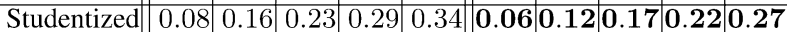

\begin{tabular}{l|l|l|l|l|l|l|l|l|l|l|l|} 
Adj Basic & $\mathbf{0 . 0 7}$ & $\mathbf{0 . 1 3}$ & $\mathbf{0 . 2 0}$ & $\mathbf{0 . 2 4}$ & $\mathbf{0 . 2 9}$ & 0.10 & 0.15 & 0.19 & 0.26 & 0.30 \\
\hline
\end{tabular} \begin{tabular}{|l|l|l|l|l|l|l|l|l|l|l|}
\hline Adj Percent. & 0.11 & 0.17 & 0.22 & 0.26 & 0.30 & 0.10 & 0.15 & 0.19 & 0.25 & 0.31 \\
\hline
\end{tabular}

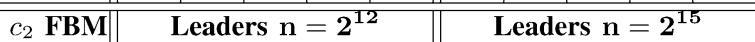
\begin{tabular}{l||c|c|c|c|c||c|c|c|c|c|c|c|c|}
$\alpha$ nominal & 0.05 & $\mathbf{0 . 1 0}$ & $\mathbf{0 . 1 5}$ & $\mathbf{0 . 2 0}$ & $\mathbf{0 . 2 5}$ & $\mathbf{0 . 0 5}$ & $\mathbf{0 . 1 0}$ & $\mathbf{0 . 1 5}$ & $\mathbf{0 . 2 0}$ & $\mathbf{0 . 2 5}$ \\
\hline
\end{tabular}

\begin{tabular}{|l||l|l|l|l|l|l|l|l|l|l|}
\hline Normal & 0.13 & 0.20 & 0.27 & 0.32 & 0.38 & 0.18 & 0.27 & 0.34 & 0.40 & 0.46 \\
\hline
\end{tabular} \begin{tabular}{|r|r|r|r|r|r|r|r|r|r|r|r|} 
Basic & 0.17 & 0.22 & 0.29 & 0.34 & 0.40 & 0.24 & 0.34 & 0.41 & 0.48 & 0.54 \\
\hline
\end{tabular} \begin{tabular}{|l|l|l|l|l|l|l|l|l|l|l|} 
Percentile & 0.14 & 0.21 & 0.28 & 0.34 & 0.40 & $\mathbf{0 . 1 2}$ & 0.21 & 0.28 & 0.34 & 0.39 \\
\hline
\end{tabular} \begin{tabular}{|l|l|l|l|l|l|l|l|l|l|l|l|l|l}
\hline Studentized & $\mathbf{0 . 1 2}$ & 0.19 & 0.26 & 0.30 & 0.34 & 0.20 & 0.30 & 0.38 & 0.44 & 0.50
\end{tabular}

\begin{tabular}{l|l|l|l|l|l|l|l|l|l|l|} 
Adj Basic & 0.14 & 0.19 & 0.22 & 0.28 & 0.34 & 0.30 & 0.33 & 0.40 & 0.46 & 0.51 \\
\hline
\end{tabular}

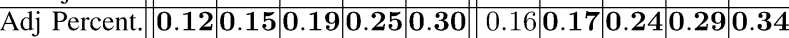

\begin{tabular}{c||c||c|}
$c_{2}$ MRW & Coefficients $\mathbf{n}=\mathbf{2}^{12}$ & Coefficients $\mathbf{n}=\mathbf{2}^{15}$
\end{tabular}

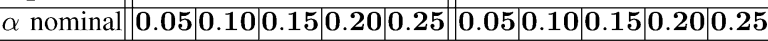

\begin{tabular}{|c||c|c|c|c|c|c|c|c|c|c|} 
Normal & $\mathbf{0 . 0 6}$ & 0.12 & 0.17 & 0.22 & 0.28 & $\mathbf{0 . 0 5}$ & $\mathbf{0 . 1 0}$ & $\mathbf{0 . 1 5}$ & $\mathbf{0 . 2 0}$ & $\mathbf{0 . 2 5}$ \\
\hline
\end{tabular} \begin{tabular}{|l|l|l|l|l|l|l|l|l|l|l|l|l|}
\hline Basic & 0.07 & 0.12 & 0.18 & 0.24 & 0.29 & $\mathbf{0 . 0 5}$ & 0.12 & 0.18 & 0.23 & 0.29 \\
\hline
\end{tabular} \begin{tabular}{l|l|l|l|l|l|l|l|l|l|l|}
\hline Percentile & 0.07 & 0.13 & 0.19 & 0.24 & 0.30 & $\mathbf{0 . 0 5}$ & $\mathbf{0 . 1 0}$ & 0.14 & 0.19 & 0.24 \\
\hline
\end{tabular} \begin{tabular}{|r||c|c|c|c|c|c|c|c|c|c|}
\hline Studentized & 0.07 & 0.12 & 0.17 & 0.23 & 0.27 & 0.04 & $\mathbf{0 . 1 0}$ & 0.16 & 0.22 & 0.28 \\
\hline
\end{tabular} \begin{tabular}{|l|l|l|l|l|l|l|l|l|l|l} 
Adj Basic & $\mathbf{0 . 0 6}$ & $\mathbf{0 . 0 9}$ & 0.14 & 0.19 & 0.24 & 0.08 & 0.13 & 0.18 & 0.24 & 0.28 \\
\hline
\end{tabular} \begin{tabular}{|l|l|l|l|l|l|l|l|l|l|l|l|}
\hline Adj Percent. & 0.07 & $\mathbf{0 . 1 1}$ & $\mathbf{0 . 1 5}$ & $\mathbf{0 . 2 0}$ & $\mathbf{0 . 2 5}$ & 0.08 & $\mathbf{0 . 1 0}$ & 0.14 & $\mathbf{0 . 2 0}$ & 0.24 \\
\hline
\end{tabular}

\begin{tabular}{|c||c||c|}
\hline$c_{2}$ MRW & Leaders $\mathbf{n}=\mathbf{2}^{12}$ & Leaders $\mathbf{n}=\mathbf{2}^{15}$ \\
\hline$\alpha$ &
\end{tabular}

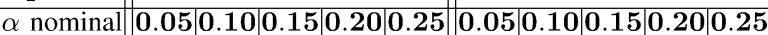

\begin{tabular}{|r||c|c|c|c|c||c|c|c|c|c|}
\hline Normal & 0.06 & 0.12 & 0.17 & 0.23 & 0.27 & 0.07 & 0.14 & 0.19 & 0.24 & 0.30 \\
\hline
\end{tabular} \begin{tabular}{l|l|l|l|l|l|l|l|l|l|l|l|}
\hline Basic & 0.10 & 0.16 & 0.21 & 0.26 & 0.31 & 0.10 & 0.18 & 0.24 & 0.30 & 0.36 \\
\hline
\end{tabular}

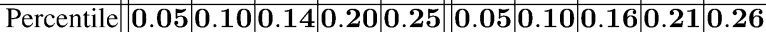
\begin{tabular}{|r||c|c|c|c|c|c|c|c|c|c|}
\hline Studentized & 0.06 & 0.11 & $\mathbf{0 . 1 6}$ & 0.21 & $\mathbf{0 . 2 5}$ & 0.06 & 0.12 & 0.18 & 0.23 & 0.28 \\
\hline
\end{tabular} \begin{tabular}{|l|l|l|l|l|l|l|l|l|l|l|} 
Adj Basic & 0.09 & 0.12 & $\mathbf{0 . 1 6}$ & 0.19 & 0.23 & 0.15 & 0.16 & 0.23 & 0.26 & 0.32 \\
\hline
\end{tabular} \begin{tabular}{|l|l|l|l|l|l|l|l|l|l|l|l|}
\hline Adj Percent. & 0.04 & 0.06 & 0.09 & 0.12 & 0.16 & 0.08 & 0.08 & $\mathbf{0 . 1 4}$ & 0.16 & 0.22 \\
\hline
\end{tabular}

the power against multiple alternatives with MRW (cf. Fig. 4), a range of ten parameter settings, $H=0.72$ and $\lambda^{2}=-c_{2}=$ $\{0.01,0.02, \ldots, 0.09,0.1\}$, is used.

\section{RESULTS}

\section{A. Significance and $p$-Value, Under $H_{0}$}

A first set of experiments is run to evaluate the actual significances and p-values of the procedures. For that, we test the hypothesis $H_{0}: c_{p}=c_{p, 0}$ when this hypothesis is true.

We obtain $N_{\mathrm{MC}}$ estimates $\hat{d}_{\alpha}$ and $\hat{p}$ for each of the proposed tests and nominal $\alpha$ 's.

Significance: The actual significances $\hat{\alpha}_{\mathrm{MC}}$ of the tests are estimated as $(\hat{\mathbb{E}}$ denotes the average performed over Monte Carlo realizations):

$$
\hat{\alpha}_{\mathrm{MC}}=\hat{\mathbb{E}}\left\{\hat{d}_{\alpha} \mid c_{p} \equiv c_{p, 0}\right\}
$$

and should ideally equal the nominal significance $\alpha$.

Table II summarizes the results for tests on $c_{2}$. We see that $\hat{\alpha}_{\mathrm{MC}}$ is in general satisfactorily close to nominal $\alpha$ for the proposed methods. For FBM, the tests employing coefficients reproduce the nominal $\alpha$ slightly better than those using Leaders, in particular for large sample size. For MRW, coefficients and Leaders-based tests perform equivalently well, both having actual significance very close to the nominal one. When using co-
TABLE III

MEAN P-VALue $\widehat{\mathbb{E}}\left\{\hat{p} \mid c_{2}=c_{2,0}\right\}$ OF BOOTSTRAP TESTS ON $c_{2}$, UsING COEFFICIENTS AND LEADERS, FOR FBM $\left(c_{2,0}=c_{2}=0\right)$ AND MRW $\left(c_{2,0}=c_{2}=-0.08\right)$. RESUlTS ClOSEST TO THE THEORETICAL Value $\mathbb{E} p=1 / 2$ ARE MARKED IN Bold

\begin{tabular}{|r||r|r||r|r||r|r||r||r||}
\hline \multicolumn{1}{|c||}{$c_{2}$} & \multicolumn{4}{c||}{ FBM } & \multicolumn{4}{c|}{ MRW } \\
\hline \hline \multicolumn{1}{|c||}{} & \multicolumn{1}{|c|}{ Coefficients } & \multicolumn{2}{c|}{ Leaders } & \multicolumn{2}{c|}{ Coefficients } & \multicolumn{2}{c|}{ Leaders } \\
\hline & $2^{12}$ & $2^{15}$ & $2^{12}$ & $2^{15}$ & $2^{12}$ & $2^{15}$ & $2^{12}$ & $2^{15}$ \\
\hline Normal & 0.43 & 0.48 & 0.42 & 0.36 & 0.47 & 0.49 & 0.49 & 0.47 \\
\hline Basic & 0.44 & 0.48 & 0.39 & 0.32 & 0.47 & 0.48 & 0.46 & 0.43 \\
\hline Percentile & 0.42 & 0.47 & 0.41 & 0.41 & 0.47 & $\mathbf{0 . 5 0}$ & $\mathbf{0 . 5 0}$ & $\mathbf{0 . 5 0}$ \\
\hline Studentized & 0.44 & $\mathbf{0 . 4 9}$ & 0.42 & 0.34 & 0.48 & 0.49 & $\mathbf{0 . 5 0}$ & 0.47 \\
\hline Adj Basic & $\mathbf{0 . 4 6}$ & 0.46 & 0.43 & 0.31 & 0.49 & 0.46 & $\mathbf{0 . 5 0}$ & 0.44 \\
\hline Adj Percent. & 0.44 & 0.46 & $\mathbf{0 . 4 7}$ & $\mathbf{0 . 4 2}$ & $\mathbf{0 . 5 0}$ & 0.49 & 0.56 & 0.52 \\
\hline
\end{tabular}
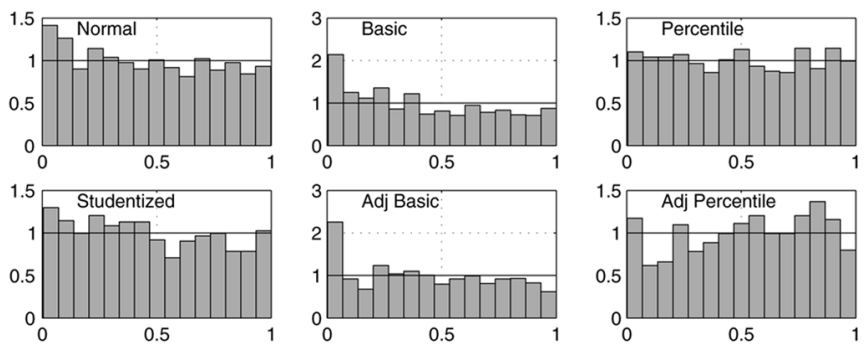

Fig. 3. Empirical distributions of the p-value of the bootstrap tests, obtained for MRW $\left(c_{2,0}=c_{2}=-0.08, n=2^{15}\right)$ using Leaders.

efficients, no clear preference can be given to any of the particular acceptance regions. We note that the adjusted percentile and the percentile (FBM), and the percentile and the studentized (MRW) method perform slightly better than the others for tests based on Leaders.

$P$-Value: Ideally, the p-value under $H_{0}$ should be uniformly distributed, with mean $1 / 2$. The average actual p-values $\hat{p}_{\mathrm{MC}}$ of the tests are estimated as $\hat{p}_{\mathrm{MC}}=\hat{\mathbb{E}}\left\{\hat{p} \mid c_{p} \equiv c_{p, 0}\right\}$ and are summarized in Table III for $c_{2}$. Examples of their empirical distributions are shown in Fig. 3. We observe that the expected uniform (mean $1 / 2$ ) distributions are satisfactorily reproduced for both FBM and MRW and for all acceptance regions. Therefore, the error rate interpretation of the estimation $\hat{p}$ of $p$ is valid for the proposed procedures. We note that whereas the adjusted methods generally improve results for small sample size $n$, it appears to be much less decisive for larger sample size. This may be due to the smaller number of double bootstrap resamples $(S=25)$ used in the latter case.

\section{B. Power Under $H_{A}$}

A second set of experiments is run to evaluate the power of the procedures. For that, we test the hypothesis $H_{0}: c_{p} \equiv 0$ when an alternative $H_{A}: c_{p}=c_{p, A} \neq 0$ is true.

Power: The actual powers of the tests are estimated as

$$
\hat{\beta}_{\mathrm{MC}}\left(c_{p, A}, \alpha\right)=\hat{\mathbb{E}}\left\{\hat{d}_{\alpha} \mid c_{p}=c_{p, A}\right\} .
$$

Table IV summarizes the results for the particular alternative $H_{A}: c_{2}=c_{2, A}=-0.08$. The larger the power, the better the test. As expected, we observe that the power increases with $n$. Also, we see that the percentile and adjusted percentile method have consistently the largest power. Whereas the tests employing coefficients achieve only low powers, the Leader-based procedures perform significantly better, with power up to 0.7 

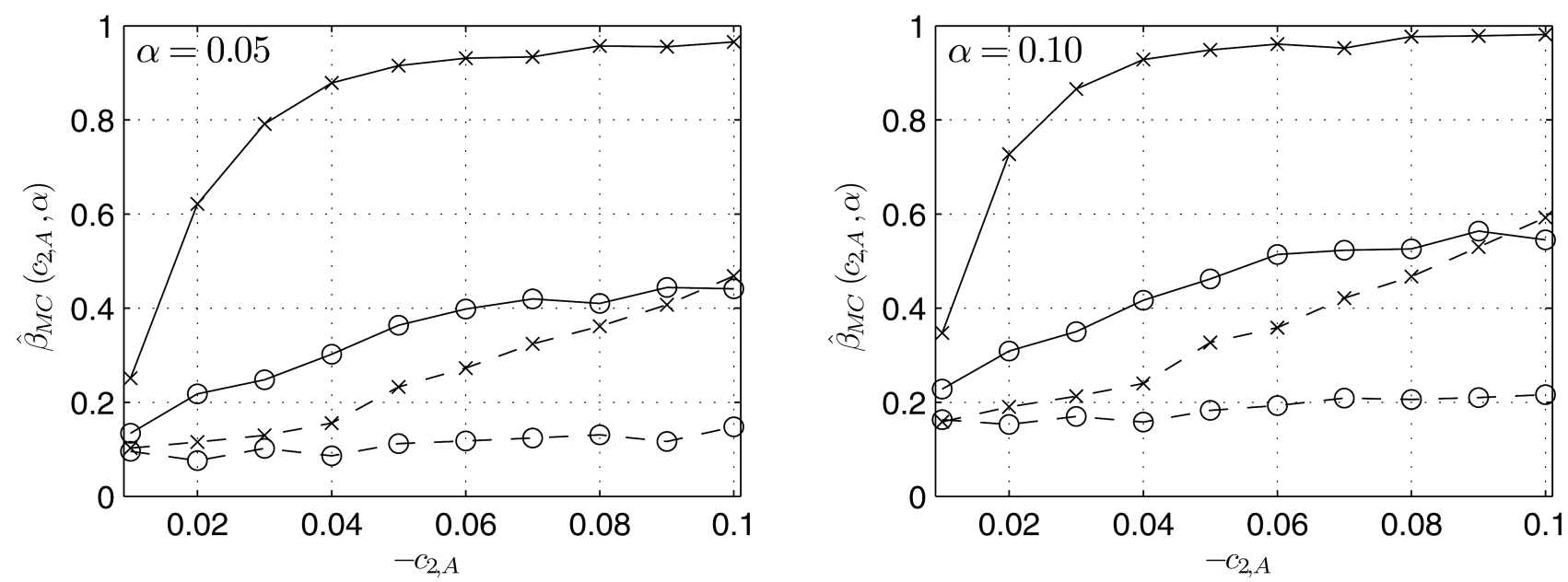

Fig. 4. Actual power $\hat{\beta}_{\mathrm{MC}}\left(c_{2}, A, \alpha\right)$ of basic bootstrap test of $H_{0}: c_{2} \equiv 0$ for MRW against various alternatives $c_{2}, A=c_{2}$, obtained for nominal significances $\alpha=0.05$ (left) and $\alpha=0.1$ (right). Dashed and solid lines represent results obtained with coefficients and Leaders, respectively. The symbols $(\mathrm{o}, \times)$ stand for $n=\left(2^{12}, 2^{15}\right)$, respectively.

TABLE IV

ACTUAL POWER $\hat{\beta}_{\mathrm{MC}}\left(c_{p, A}, \alpha\right)$ VERSUS SIGNIFICANCE OF BOOTSTRAP TESTS OF $H_{0}: c_{2} \equiv 0$ FOR MRW $\left(c_{2, A}=c_{2}=-0.08\right)$, USING COEFFICIENTS (TOP) AND LEADERS (BOTTOM). BEST RESUlTS ARE MARKED IN BOLD

\begin{tabular}{|r||c|c|c|c|c|c||c|c|c|c|c|}
\hline$c_{2}$ MRW & \multicolumn{3}{|c|}{ Coefficients $\boldsymbol{n}_{\mathbf{2}}^{\mathbf{1 2}}$} & \multicolumn{4}{|c|}{ Coefficients $\boldsymbol{n = 2}$} \\
\hline$\alpha$ nominal & 0.05 & 0.10 & 0.15 & 0.20 & 0.25 & 0.05 & 0.10 & 0.15 & 0.20 & 0.25 \\
\hline \hline Normal & 0.16 & 0.25 & 0.31 & 0.38 & 0.42 & 0.45 & 0.55 & 0.62 & 0.67 & 0.71 \\
\hline Basic & 0.13 & 0.21 & 0.28 & 0.35 & 0.40 & 0.40 & 0.50 & 0.57 & 0.62 & 0.66 \\
\hline Percentile & $\mathbf{0 . 2 2}$ & $\mathbf{0 . 3 0}$ & $\mathbf{0 . 3 6}$ & $\mathbf{0 . 4 1}$ & $\mathbf{0 . 4 7}$ & 0.48 & 0.59 & $\mathbf{0 . 6 6}$ & $\mathbf{0 . 7 1}$ & $\mathbf{0 . 7 5}$ \\
\hline Studentized & 0.13 & 0.18 & 0.25 & 0.32 & 0.37 & 0.29 & 0.41 & 0.50 & 0.57 & 0.61 \\
\hline Adj Basic & 0.12 & 0.18 & 0.24 & 0.30 & 0.35 & 0.46 & 0.53 & 0.58 & 0.63 & 0.66 \\
\hline Adj Percent. & 0.19 & 0.27 & 0.33 & 0.38 & 0.43 & $\mathbf{0 . 5 5}$ & $\mathbf{0 . 6 0}$ & $\mathbf{0 . 6 6}$ & $\mathbf{0 . 7 1}$ & 0.74 \\
\hline \hline$c_{2}$ MRW & \multicolumn{6}{|c||}{ Leaders } & $\boldsymbol{n}=\mathbf{2}^{\mathbf{1 2}}$ & \multicolumn{5}{|c|}{ Leaders } & $\boldsymbol{n}=\mathbf{2}^{\mathbf{1 5}}$ \\
\hline$\alpha$ nominal & 0.05 & 0.10 & 0.15 & 0.20 & 0.25 & 0.05 & 0.10 & 0.15 & 0.20 & 0.25 \\
\hline \hline Normal & 0.61 & 0.71 & 0.78 & 0.82 & 0.85 & 0.97 & $\mathbf{0 . 9 9}$ & $\mathbf{0 . 9 9}$ & 0.99 & 0.99 \\
\hline Basic & 0.53 & 0.63 & 0.69 & 0.74 & 0.78 & 0.96 & 0.97 & 0.98 & 0.99 & 0.99 \\
\hline Percentile & $\mathbf{0 . 7 0}$ & $\mathbf{0 . 8 0}$ & $\mathbf{0 . 8 4}$ & $\mathbf{0 . 8 7}$ & $\mathbf{0 . 8 9}$ & 0.98 & $\mathbf{0 . 9 9}$ & $\mathbf{0 . 9 9}$ & $\mathbf{1 . 0 0}$ & $\mathbf{1 . 0 0}$ \\
\hline Studentized & 0.45 & 0.56 & 0.62 & 0.67 & 0.71 & 0.90 & 0.94 & 0.96 & 0.97 & 0.98 \\
\hline Adj Basic & 0.49 & 0.56 & 0.61 & 0.67 & 0.71 & 0.97 & 0.97 & 0.98 & 0.98 & 0.99 \\
\hline Adj Percent. & 0.66 & 0.71 & 0.76 & 0.81 & 0.84 & $\mathbf{0 . 9 9}$ & $\mathbf{0 . 9 9}$ & $\mathbf{0 . 9 9}$ & 0.99 & 0.99 \\
\hline
\end{tabular}

for small sample size and $\alpha$, and approximately 1 for large sample size. The superiority of the Leaders-based procedures is also clearly illustrated in Fig. 4, which shows the power of the basic bootstrap test (with $R=199$ ) for a set of alternatives $c_{2}=c_{2, A}=\{-0.01,-0.02, \ldots,-0.1\}$; whereas the coefficient-based test achieves only low powers over the whole range of alternatives, the Leader-based procedure maintains large powers over a wide range of alternatives. The powers of the Leader-based test remain significantly above those of the coefficient-based test for alternatives close to the null value $c_{2,0}=0$, in particular for large sample size.

\section{Conclusion}

The results discussed above show that the proposed non parametric bootstrap procedures for testing $H_{0}: c_{p}=c_{p, 0}$ present satisfactory performance in reproducing the targeted significances and $\mathrm{p}$-values equivalently for wavelet coefficients and wavelet Leaders. However, the test procedures involving Leaders are significantly more powerful than their coefficient-based counterparts and are thus clearly preferable. The choice of acceptance region has little impact on the actual significances $\hat{\alpha}_{\mathrm{MC}}$ and on the empirical distributions of the p-values, with a slight preference, however, for the percentile and adjusted percentile methods. These methods obtain as well the largest powers; hence, they will be preferred. Furthermore, the adjusted method requires the calculation of double bootstrap resamples, increasing the computational cost for the bootstrap by a factor $S$, without bringing significant improvements. For instance, on a standard PC, the bootstrap estimation and test procedures for $c_{1}, c_{2}$ for a single observation of length $n=2^{12}\left(2^{15}\right)$ requires around $1.6(2.7) \mathrm{s}$ for simple bootstrap methods and 21.3(48.4)s for double bootstrap methods ( $R=399, S=25$ in all cases). It is possible that the results obtained with double bootstrap methods could be slightly improved by using a larger number of double bootstrap resamples $S$, however at the cost of further increasing computational load considerably.

Therefore, we conclude that tests for $H_{0}: c_{p}=c_{p, 0}$ should be based on Leaders and percentile acceptance regions.

\section{Further Developments}

The bootstrap tests were illustrated here with MRW, a LogNormal multifractal process (i.e., $\forall p \geq 3, c_{p}=0$ ). However, as mentioned above, the tests can be applied to any multifractal process and any $c_{p}$. For instance, applying the tests with the choice $H_{0}: c_{3}=c_{3,0} \equiv 0$ provides us with indications to decide whether a Log-Normal multifractal process or a more sophisticated MF model is to be used to describe the data under study. Let us recall that, in itself, $c_{3}=0$ does not prove that data follow a Log-Normal process; however, this is a very valuable and practically useful information. We have studied the relevance and statistical performance of such tests through numerical simulations for another class of synthetic multifractal processes: compound Poisson cascades [3], whose $c_{3} \neq 0$ can be set $a$ priori. For space reasons, such results are not presented here and will be reported elsewhere. 

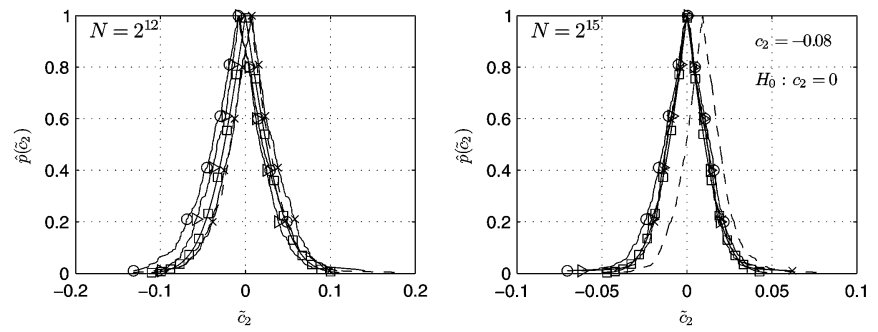

Fig. 5. P-value function estimate from Monte Carlo simulation (dashed) and bootstrap estimates from single realization (solid) versus a potentially observable value $\tilde{c}_{2}$, obtained for MRW ( $\left.c_{2}=-0.08, c_{2,0}=0\right)$ using Leaders. The symbols $(\square, \triangleright, \times, \circ)$ stand for (Normal, Basic, Percentile, Studentized) acceptance regions, respectively.

Along another line, alternative test statistics such as $T=$ $\left|c_{p}-c_{p, 0}\right|$ have also been studied. Results on this, yielding similar conclusions, are not reported here.

\section{CONCLUSION AND PERSPECTIVES}

Practical Test Procedure: We have constructed a practical procedure that enables us to test a given a priori chosen multifractal property: $H_{0}: c_{p}=c_{p, 0}$. Obviously, the choice $H_{0}$ : $c_{2}=c_{2,0} \equiv 0$ can be seen as a test of mono- versus multifractality (indeed, it is conjectured, that $c_{2}=0 \Rightarrow \forall p \geq 3, c_{p}=$ 0 ). We showed from numerical simulations on synthetic multifractal processes that such tests possess satisfactory statistical performance. A Matlab procedure, designed by the authors, implements this proposed multifractality test procedures. To the best of our knowledge, this is the first and only practical multifractal test that can actually be applied to a single observation of data with finite length. We see this result as an important contribution to empirical multifractal analysis.

In addition to obtaining $\hat{d}_{\alpha}$ and $\hat{p}$, our practical test procedure also outputs, from a single realization, an approximate $\mathrm{p}$-value as a function of a potentially observable value $\tilde{c}_{p}$. This is done by (numerically) inverting the estimated null distribution

$$
\hat{p}\left(\tilde{c}_{p}\right)=2 \cdot \min \left(\hat{P}_{0}^{T}(\gamma) ; 1-\hat{P}_{0}^{T}(\gamma)\right)
$$

with $\gamma=\tilde{c}_{p}-c_{p, 0}$ for test statistic $T_{B}$, and $\gamma=\left(\tilde{c}_{p}-c_{p, 0}\right) / \hat{\sigma}^{*}$ for $T_{S}$. Examples of such p-value functions are depicted in Fig. 5, together with an estimate from MC simulation. For all acceptance region methods, we observe that the functions $\hat{p}\left(\tilde{c}_{p}\right)$ match satisfactorily well the one obtained from MC simulations. Such p-value functions can be seen as a powerful help for the practitioner. Indeed, the narrower the functions, the more powerful the tests.

When analyzing real data, power functions such as those proposed in Fig. 4 can usefully complete the test procedure. They can be estimated by numerical simulations on synthetic multifractal processes whose parameters and size fit those of the data under analysis.

Perspectives: Parametric bootstrap tests can also be used. They must be based on the assumption that the distributions of wavelet Leaders can be modeled via a general class of functions. This is under current investigations. Methods for estimating the power of a test against specific alternatives from a single realization can further improve the practical test procedures and are currently tested.

There are major potential interests in applying these multifractal tests to empirical data from hydrodynamic turbulence, computer network traffic and biomedical applications. We are currently analyzing such data.

\section{REFERENCES}

[1] P. Abry, P. Flandrin, M. Taqqu, and D. Veitch, "Wavelets for the analysis, estimation and synthesis of scaling data," in Self-Similar Network Traffic and Performance Evaluation. New York: Wiley, Spring 2000.

[2] E. Bacry, J. Muzy, and A. Arneodo, "Singularity spectrum of fractal signals from wavelet analysis: Exact results," J. Stat. Phys., vol. 70, pp. 635-674, 1993.

[3] J. Barral and B. Mandelbrot, "Multiplicative products of cylindrical pulses," Probab. Theory Relat. Fields, vol. 124, pp. 409-430, 2002.

[4] S. Jaffard, M. Lapidus and M. van Frankenhuijsen, Eds., "Wavelet techniques in multifractal analysis," in Fractal Geometry and Applications: A Jubilee of Benoît Mandelbrot, Proc. Symposia in Pure Mathematics, 2004, vol. 72, no. 2, pp. 91-152, AMS.

[5] S. Jaffard, B. Lashermes, and P. Abry, "Wavelet leaders in multifractal analysis," in Wavelet Analysis and Applications, T. Qian, M. I. Vai, and X. Yuesheng, Eds. Basel, Switzerland: Birkhäuser Verlag, 2006, pp. 219-264.

[6] H. Wendt, S. G. Roux, and P. Abry, "Bootstrap for log wavelet leaders cumulant based multifractal analysis," presented at the EUSIPCO, Florence, Italy, Sep. 4-8, 2006.

[7] B. Castaing, Y. Gagne, and M. Marchand, "Log-similarity for turbulent flows," Physica D, vol. 68, pp. 387-400, 1993.

[8] E. Bacry, J. Delour, and J. F. Muzy, "Multifractal random walk," Phys. Rev. E, vol. 64, p. 026103, 2001.

[9] B. Efron, The Jackknife, the Bootstrap, and Other Resampling Plans. Philadelphia, PA: SIAM, 1982.

[10] A. M. Zoubir and D. R. Iskander, Bootstrap Techniques for Signal Processing. Cambridge, U.K.: Cambridge Univ. Press, 2004.

[11] A. M. Zoubir, "On confidence intervals for the coherence function," in Proc. 30th IEEE Int. Conf. Acoustics, Speech, Signal Processing (ICASSP), Philadelphia, PA, Mar. 18-23, 2005, pp. 413-416.

[12] A. C. Davison and D. V. Hinkley, Bootstrap Methods and Their Application, ser. Statistical and Probabilistic Mathematics. Cambridge: Cambridge Univ. Press, 1997.

[13] D. B. Percival, S. Sardy, and A. C. Davison, "Wavestrapping time series: Adaptive wavelet-based bootstrapping," in Nonlinear and Nonstationary Signal Processing, W. J. Fitzgerald, R. L. Smith, A. T. Walden, and P. C. Young, Eds. Cambridge, U.K.: Cambridge Univ. Press, 2000, pp. 442-471.

[14] A. M. Sabatini, "Wavelet-based estimation of $1 / f$-type signal parameters: Confidence intervals using the bootstrap," IEEE Trans. Signal Process., vol. 47, no. 12, pp. 3406-3409, 1999.

[15] H. Wendt and P. Abry, "Bootstrap for multifractal analysis," in Proc. IEEE Int. Conf. Acoustics, Speech, Signal Processing (ICASSP), Toulouse, France, May 14-19, 2006, pp. 812-815.

[16] I. Daubechies, Ten Lectures on Wavelets. Philadelphia, PA: SIAM, 1992.

[17] S. Mallat, A Wavelet Tour of Signal Processing. San Diego, CA: Academic Press, 1998.

[18] J. F. Muzy, E. Bacry, and A. Arneodo, "The multifractal formalism revisited with wavelets," Int. J. Bifurc. Chaos, vol. 4, no. 2, pp. 245-301, 1994.

[19] P. Gonçalvès and R. H. Riedi, "Wavelet analysis of fractional brownian motion in multifractal time," in Proc. 17th Colloquium GRETSI, Vannes, France, Sep. 13-17, 1999, pp. 23-26.

[20] R. H. Riedi, "Multifractal processes," in Long-Range Dependence: Theory and Applications, Doukhan, Oppenheim, and Taqqu, Eds. Basel, Switzerland: Birkhäuser, 2002, pp. 625-715.

[21] V. J. Ribeiro, R. H. Riedi, and R. G. Baraniuk, "Wavelets and multifractals for network traffic modeling and inference," presented at the IEEE Int. Conf. Acoustics, Speech, Signal Processing (ICASSP), Salt Lake City, UT, May 7-11, 2001.

[22] U. Frisch and G. Parisi, "Fully developed turbulence and intermittency," in Proc. Int. Summer School Turbulence Predictability in Geophysical Fluid Dynamics Climate Dynamics, 1985, pp. 84-88. 
[23] S. Jaffard, "Multifractal formalism for functions, Part 2: Self-similar functions," SIAM J. Math. Anal., vol. 28, no. 4, pp. 971-998, 1997.

[24] R. H. Riedi, "An improved multifractal formalism and self-similar measures," J. Math. Anal. Appl., vol. 189, pp. 462-490, 1995.

[25] J. Delour, J. F. Muzy, and A. Arneodo, "Intermittency of 1d velocity spatial profiles in turbulence: A magnitude cumulant analysis," Euro. Phys. J. B, vol. 23, pp. 243-248, 2001.

[26] M. Kendall and A. Stuart, The Advanced Theory of Statistics, Distribution Theory. London, U.K.: Griffin, 1977, vol. 1.

[27] E. L. Lehmann, Testing Statistical Hypotheses. New York: Wiley, 1959.

[28] P. Hall and S. R. Wilson, "Two guidelines for bootstrap hypothesis testing," Biometrics, vol. 47, no. 2, pp. 757-762, 1991.

[29] G. Samorodnitsky and M. Taqqu, Stable Non-Gaussian random processes. New York: Chapman and Hall, 1994.

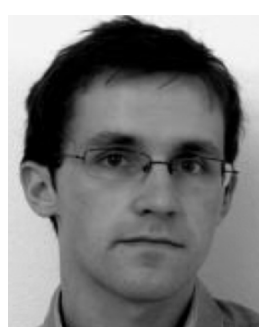

Herwig Wendt (M'05) was born in Steyr, Austria, in 1979. He received the M.S. degree in electrical engineering and telecommunications from Vienna University of Technology, Austria, in 2005. He is currently working towards the Ph.D. degree in physics and signal processing at the Laboratoire de Physique at Ecole Normale Supérieure de Lyon, France.

His research interests include scale invariance phenomena, point processes, extreme values, bootstrap methods, and machine learning.

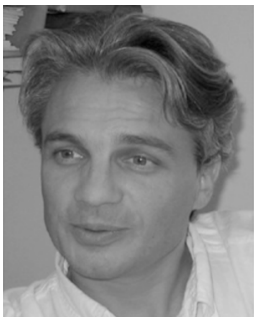

Patrice Abry was born in Bourg-en-Bresse, France, in 1966. He received the Professeur-Agrégé de Sciences Physiques degree from the Ecole Normale Supérieure de Cachan, France, in 1989 and the Ph.D. degree in physics and signal processing from the Ecole Normale Supérieure de Lyon and Université Claude-Bernard Lyon I, France, in 1994.

Since October 1995, he has been a permanent CNRS Researcher at the Laboratoire de Physique of Ecole Normale Supérieure de Lyon. His current research interests include wavelet-based analysis and modeling of scaling phenomena and related topics (self-similarity, stable processes, multifractal, 1/f processes, long-range dependence, local regularity of processes, infinitely divisible cascades, departures from exact scale invariance, etc.). The main applications under current investigation are hydrodynamic turbulence and the analysis and modeling of computer network teletraffic. He is also involved in the study of baroreflex sensitivity with a French medical group at University Claude Bernard Lyon I. He recently started a wavelet-based detection/analysis of acoustic gravity waves in the ionosphere. He is the author of a book Ondelettes et Turbulences-Multirésolution, Algorithmes de Décompositions, Invariance d'Échelle et Signaux de Pression (Paris, France: Diderot, Éditeur des Sciences et des Arts, 1997). He is also the coeditor of a book in French entitled Lois d'Échelle, Fractales et Ondelettes (Paris, France: Hermès, 2002).

Dr. Abry received the AFCET-MESR-CNRS prize for best $\mathrm{Ph}$.D. dissertation on signal processing for 1993-1994 